James D. Gounder, Peter Kutne, Wolfgang Meier, Development of a laserinduced plasma probe to measure gas phase plasma signals at high pressures and temperatures, Spectrochemica Acta Part B 74-75 (2012) 66-73.

The original publication is available at www.elsevier.com

http://dx.doi.org/10.1016/j.sab.2012.06.018 


\title{
Development of a Laser-induced Plasma Probe to Measure Gas Phase Plasma Signals at High Pressures and Temperatures
}

\author{
J.D. Gounder ${ }^{\mathrm{a}, *}$, P. Kutne ${ }^{\mathrm{a}}$, W. Meier ${ }^{\mathrm{a}}$ \\ ${ }^{a}$ Deutsches Zentrum für Luft- und Raumfahrt, Institut für Verbrennungstechnik, Pfaffenwaldring 38-40, 70569 Stuttgart, \\ Germany
}

\begin{abstract}
The ability of Laser Induced Breakdown Spectroscopy (LIBS) technique for on line simultaneous measurement of elemental concentrations has led to its application in a wide number of processes. The simplicity of the technique allows its application to harsh environments such as present in boilers, furnaces and gasifiers. This paper presents the design of a probe using a custom optic which transforms a round beam into a ring (Donut) beam, which is used for forming a plasma in an atmosphere of nitrogen at high pressure $(20 \mathrm{bar})$ and temperature $\left(200{ }^{\circ} \mathrm{C}\right)$. The LIBS experiments were performed using a high pressure cell to characterize and test the effectiveness of the donut beam transmitted through the LIBS probe and collect plasma signal in back scatter mode. The first tests used the second harmonic of a Nd:YAG laser, pulse width $7 \mathrm{~ns}$, to form a plasma in nitrogen gas at five different pressures $(1,5,10,15$ and 20 bar) and three different gas temperatures $\left(25,100\right.$ and $\left.200{ }^{\circ} \mathrm{C}\right)$. The uniqueness of this probe is the custom made optic used for reshaping the round laser beam into a ring (Donut) shaped laser beam, which is fed into the probe and focused to form a plasma at the measurement point. The plasma signal is collected and collimated using the laser focusing lens and is reflected from the laser beam axis onto an achromatic lens by a high reflection mirror mounted in the center section of the donut laser beam. The effect of gas pressure and temperature on N(I) lines in the high pressure cell experiment shows that the line intensity decreases with pressure and increase with temperature. Mean plasma temperature calculated using the ratios of N(I) line intensities range from $7400 \mathrm{~K}$ to $8900 \mathrm{~K}$ at 1 bar and $2400 \mathrm{~K}$ to $3200 \mathrm{~K}$ at 20 bar for the three different gas temperatures. The results show that as a proof of principle the donut beam optics in combination with the LIBS probe can be used for performing extensive LIBS measurements in well controlled laboratory environment as well as harsh and demanding environments of practical devices at both high pressures and temperatures.
\end{abstract}

Keywords: LIBS, High Pressure, High Temperature, Nitrogen

\section{Introduction}

The reduction of $\mathrm{CO}_{2}$ emissions has become a major topic of research in the last decade due to the negative environmental impact of $\mathrm{CO}_{2}$ and other green house gases on world climate.

${ }^{*}$ Corresponding author

Email address: James.Gounder@dlr.de (J.D. Gounder)

Preprint submitted to Spectrochimica Acta Part B: Atomic Spectroscopy

June 11, 2012 
In 2007 the European Commission has declared to reduce the emission of greenhouse gases by $20 \%$ until 2020 and increase the use of renewable energy [1]. The fossil fuels will remain the largest source for energy conversion in the next decades. Thus there is an increased interest in power generation industry towards technologies, which help to reduce $\mathrm{CO}_{2}$ emissions from fossil fuels. One of the these technologies involve the Integrated Gasification Combined Cycle (IGCC) power plant. While gasifiers for other applications already exist [2]. In order to be used in an IGCC the gasifier has to be optimized regarding efficiency and reliability. Slagging caused by metals and other inorganics in the combustion products of coal has been a major factor affecting the efficiency of the gasifiers.

A lot of challenges exist in order to perform measurements in gasifiers to study the gasification process as well as measure impurities present in the product gas. One technique that could be used for studying and performing measurements in gasifiers would be the laser-induced breakdown spectroscopy (LIBS) technique. The LIBS consist of a high energy laser pulse with a short pulse width, focused onto a sample. Photon absorption causes vaporisation of the sample material resulting in a plasma with a temperature of several thousands of Kelvins, which dissociates the material into atoms, electrons and ions. As the plasma temperature and electron density decay, the species present in the plasma emit photons at characteristic atomic frequencies as they relax back to their ground state. Time gated detection of the emitted photons using a spectrograph coupled with suitable calibration allows the identification and quantification of elemental concentrations.

The development of LIBS technique in last two decades alone has seen the LIBS technique receive considerable attention in fundamental studies as well as applications to practical systems $[3,4,5]$. The use of a laser and a focusing lens for forming a plasma and a spectrograph equipped with a intensified charge coupled device (CCD), for detecting the signal from the plasma makes the LIBS technique a very useful analytical tool for elemental analysis. The simplicity and versatility of the LIBS measurement technique and minimum effort required for sample preparation allows online, multi elemental detection of solids, liquids and gases. Cremers and Chinni [4] and the references within have listed a large number of applications where LIBS measurement technique is used. The applications which relate to the work done in this paper are the analysis of impurities in gases $[6,7,8]$, measurements of equivalence ratio and fuel concentrations $[9,10,11]$ and development of probes for measurements in gasifiers, furnaces and boilers $[12,13,14,15]$.

Blevins et al [12] and Molina et al [13] have performed LIBS experiment in high temperature environment with promising results. They have also highlighted difficulties encounted while performing measurements in boilers and furnaces, especially with the layout and setup of the measurement and probe. Similar challenges can be expected in gasifiers. Eseller et al [8] have performed LIBS measurement to detect impurities of $\mathrm{N}_{2}$, Ar and $\mathrm{O}_{2}$ in $\mathrm{H}_{2}$ and study the effect of pressure on the measurements. Their experiments were carried out at pressures going up to 5 bar. The work of Blevins et al [12] also involved the development of a measurement probe to be used in the boiler facility. Work has also been done in the development of fiber probes by Dumitrescu et el $[16,17]$ for performing LIBS measurements in gases. These studies have provided good insight into design and development of LIBS probes.

This paper presents the development of a laser beam transforming optics to be used in combination with the LIBS probe for measurements to be performed at high pressure and temperature environment. The LIBS probe has been designed for measurements to be performed at a gasifier facility where the initial condition of the gas at the measurement point can be around $300{ }^{\circ} \mathrm{C}$ and over 20 bar pressure. A special custom made optic is used for transforming the $532 \mathrm{~nm}$ round beam from a Nd:YAG laser into a donut shaped beam, which is then transmitted through the 
probe and focused to form a plasma. The plasma signal is collected in the back scatter mode through the laser focusing lens and a high reflective mirror in the center of the donut beam forming optics. A comparison between plasma formed using a donut beam profile and a round beam profile as well as the signal collected is presented.

The similarity in qualitative trends shown in the plasma signal from a round beam and the donut beam provides confidence in using the LIBS probe with donut forming optics and collection in back scatter mode. So far to the best of the authors knowledge, availability of data from LIBS measurements or studies performed in gases at high pressures ( $>10$ bar) and high temperatures are limited. This paper also provides the characterization of the plasma formed in nitrogen gas at three different gas temperatures and five different gas pressures using the LIBS probe.

\section{Experimental Method}

A number of tests have been carried out to investigate the effectiveness of the high pressure LIBS probe in transmitting a laser beam and collecting plasma signal in back scatter mode. This section provides detailed description of the probe, the experimental setup and measurement conditions. The use of a high pressure cell allowed for experiments to be performed in a controlled environment, eliminating problems that may arise at the high pressure test rig and provided a much better control of the boundary conditions to study the effect of pressure and temperature on the plasma. Detailed experiments to study the behavior of the laser plasma formed in nitrogen gas atmosphere at 5 different pressures and 3 different temperatures were performed using the LIBS probe and the high pressure cell.

\subsection{LIBS Probe}

The main purpose of the high pressure LIBS probe is to be used for performing LIBS measurements at a gasifier facility hence the probe was designed to be operated in conditions with pressures up to 40 bar and temperatures reaching $1600{ }^{\circ} \mathrm{C}$. Figure 1 shows a cross sectioned sketch of the probe. The probe contains a standard DN50 flange which allows simple adapters to be used for mounting the probe to measurement access ports available at the gasifier facility as well as the test rig at DLR. For measurements at high temperature the probe requires water cooling. Two quartz windows are used for sealing the inlet and exit end of the probe with provision for purge air cleaning for the exit window. A best form lens of diameter $25 \mathrm{~mm}$ and focal length $(f)$ of $100 \mathrm{~mm}$ is mounted inside the probe as marked in Fig. 1.

To transmit a $532 \mathrm{~nm}$ round laser beam through the LIBS probe to form plasma and collect the signal in back scatter required a custom made beam splitter (filter), that reflected the laser light at $532 \mathrm{~nm}$ and transmitted the plasma signal in the spectral range 350-700 $\mathrm{nm}$. In order to avoid interference from the scattered green light, the beam splitter had a $80 \mathrm{~nm}$ bandwidth (center $\lambda=532 \mathrm{~nm}$ ) notch in the transmission spectral range. This transmission efficiency of the beam splitter dropped significantly at lower wavelengths. A number of other filters were also tested including a standard notch filter but all filters had significant reduction in transmission at lower wavelengths. Due to reduced detectable spectral range and transmissibility of the beam splitter and standard filters, a new set of optics were designed to enable transmission of a $532 \mathrm{~nm}$ beam through the LIBS probe, as well as allow collection of the plasma signal without the need of a filter.

This new optical component was designed specifically for the LIBS probe, for transforming a round beam profile into a ring (donut) shaped beam profile. The hole in the middle of the donut 
beam provided allowance for a high reflective mirror component to be mounted for reflecting the plasma signal $90^{\circ}$ from the laser axis to the collection optics comprising of an achromatic lens and fiber coupler. These optics are assembled and aligned to the optical axis of the probe, at a distance of $600 \mathrm{~mm}$, from input window of the probe. The donut laser beam profile is fed into the probe and focused by the best form lens creating a plasma. The signal from the plasma is collected in the back scatter mode. The insert in Fig. 1 a) best illustrates the transformation of the laser beam and the collection of the signal. Figure $1 \mathrm{~b}$ ) shows the LIBS probe assembled at the high pressure rig at DLR. Details about the high pressure rig can be found in [18].

\subsection{Laser configuration and experimental setup}

The second harmonic (532 nm) of a Q-switched Nd:YAG laser (Innolas spitlight 600) operating at a repetition rate of $10 \mathrm{~Hz}$ and with a pulse duration of $7 \mathrm{~ns}$ was used for all experiments. Figure 2 shows the layout of the laser setup to form plasma inside the high pressure cell and the collection optics. The initial beam diameter of $6 \mathrm{~mm}$ out of the laser is expanded and directed into the beam transforming optics using a beam splitter which reflects $95 \%$ of the laser energy and transmits 5\% into a pyroelectric detector used for monitoring the laser energy on a shot to shot basis. The donut beam is directed into the probe and is focused by a best form lens of $100 \mathrm{~mm}$ focal length to form a plasma inside the high pressure cell. The transmission efficiency of the beam transforming optics is $75 \%$. The mean laser energy in the donut beam was around $250 \mathrm{~mJ}$. A LaVision imager intense camera mounted at $90^{\circ}$ is used for imaging the plasma. The light from the plasma is attenuated using neutral density filters to avoid damaging the CCD chip.

Spectral emission from the laser induced plasma was collected via back scatter by a high reflective metal mirror mounted in the center of the laser profile. This is shown in the insert in Fig. 1 a). The reflected signal is focused by a achromatic lens and is collected by a fiber optics coupler connected to a echelle spectrograph (LLA Instruments ESA 4000) via a optical fiber. The spectrograph has a built in ICCD detector. The image intensifier has been optimised for maximum sensitivity at $450 \mathrm{~nm}$. The spectral resolution of the echele spectrograph used in these experiments, ranges from $5 \mathrm{pm}(200 \mathrm{~nm})$ to $19 \mathrm{pm}(780 \mathrm{~nm})$. The computer controlling the spectrograph system, is equipped with a fast pulse generator and is programmed to control the laser and the pyroelectric detector. Esawin software (LLA Instruments) is used for collecting the spectrum. Further detailed description of the spectrograph hardware can be found in [19]. The gate width for the intensifier was set to $10 \mu \mathrm{s}$. For each gas pressure and temperature condition 100 spectra were collected and each spectrum was an accumulation of 10 laser shots before the CCD was readout. The laser energy was also, recorded for each readout, which is the mean of 10 laser shots.

\subsection{Measurement conditions}

The high pressure measurements were performed in a laboratory setup using a cell, which can be filled with a gas, with provision to pressurize and heat the gas. The external dimensions of the high pressure cell is $70 \mathrm{~mm} \times 70 \mathrm{~mm} \times 65 \mathrm{~mm}$ with four access windows as shown in Fig. 2 . It was used for characterizing the plasma formation at high pressures and temperatures. In this experiment the LIBS probe was at atmospheric condition but was still used for transmitting the laser to form the plasma inside the cell and collect the signal in back scatter mode. Nitrogen was fed through the cell and the gas pressure was regulated using a needle valve and monitored using a pressure gauge connected to the output line of the cell. The cell is fitted with heating elements which allows the cell and the gas flowing through it, to be heated. Thermocouples 
are used for monitoring the gas temperature. Figure 3 shows the variation in the density of nitrogen gas with pressure and temperature. Plasma is formed in pure nitrogen atmosphere at the given gas pressures and temperatures as shown in Fig. 3. A second set of measurement was performed where the collection optics were placed at the second viewing window opposite the CCD camera. Here the laser beam transforming optic was replaced with a mirror to direct an expanded round beam into the probe. Measurements were performed for the initial conditions presented in Fig. 3.

\section{Results}

\subsection{High pressure test cell results}

The plasma formed in the nitrogen gas inside the high pressure cell was imaged using a LaVision imager intense CCD camera. Hundred single shot images were collected and each image was corrected for background noise and normalized by the attenuation factor from the neutral density filters. The average of the 100 images collected for plasma formed in nitrogen at three temperatures and five pressures was calculated for two different initial beam shapes. The first set of images were collected for an initial beam profile having a donut shape and the second set with a round beam profile. Figure 4 shows the mean images of the plasma formed with a donut (a) and a round (b) beam profile.

The single image size is $12 \mathrm{~mm}(\mathrm{~W}) \times 7 \mathrm{~mm}(\mathrm{H})$ and the color scale representing the magnitude of light intensity in counts is 0 for black and maximum (65000 counts) is represented by white. The average laser energy in the donut beam was $250 \mathrm{~mJ} /$ pulse whereas the round beam had $320 \mathrm{~mJ} /$ pulse. It should be noted that the initial laser energy input into the donut forming optics was $320 \mathrm{~mJ} /$ pulse. Hence for the round beam experiment as stated earlier the donut forming optics were replaced with a standard high reflective mirror. The donut beam had a $20 \mathrm{~mm}$ outside diameter at the entrance window of the probe whereas the diameter of the round beam was $15 \mathrm{~mm}$. Assuming that both beams behave as a Gaussian beam then the spot size at the focal position can be calculated using $r=\lambda \mathrm{f} / \pi \mathrm{r}_{o}$, where $\mathrm{f}$ is the focal distance, $\mathrm{r}$ is the beam radius and subscript $o$ represents the beam radius at the focal position. It should be noted that the assumption that donut beam behaves as a Gaussian beam is very crude but this does give an approximate size of the spot diameter which is acceptable for the purpose of this study. The focal spot diameter is $3.4 \mu \mathrm{m}$ and $4.5 \mu \mathrm{m}$ for the donut and the round beam respectively. The diffraction limited spot size diameter is $3.2 \mu \mathrm{m}$ for the focusing lens setup used for the probe.

The plasma formed using the donut beam profile looks more stable and uniform in shape compared to the plasma formed using the round beam. It should be noted that the laser energy per pulse for the round beam is $25 \%$ higher and the focal spot area is $75 \%$ bigger if the beams behaves as a Gaussian beam. If the diffraction limited spot diameter is considered then the round beam will have a higher irradiance at the focal spot which could be why the plasma images from the round beam show non uniform plasma bubbles. The change in plasma shape and size at different gas pressures and temperatures could be due to the effect of the gas density on the formation of the plasma. The effect of gas density is transferred into focusing of the laser beam through variation of the refractive index of the gas. These images do not provide any spectral information but it does give an insight into the form of the plasma and its reproducibility, especially at high pressure.

The entire experimental setup had to be triggered by the spectrograph computer mainly due to the laser energy monitoring pyroelectric detector functioning only with the internal triggering 
from the spectrograph computer. This meant that the minimum gate delay time could be set to the minimum gate time allowed by the esawin software, which is $1 \mu \mathrm{s}$. A preliminary test was conducted where plasma signal in air was measured at five different pressures. This test included varying the gate width to optimise the signal to background ratio. This test provided the optimum delay time of $10 \mu \mathrm{s}$. As stated earlier each measured spectrum is an accumulation of 10 laser shots before the spectrum is read out. Spectra are measured with a gate delay of $1 \mu \mathrm{s}$ after the q-switch is triggered with an intensifier gate width of $10 \mu \mathrm{s}$.

The raw data is exported to be post processed in Matlab 2007 software. The measured spectra are spectrally corrected using the measured spectral response function of the entire optical path. Each spectrum is also corrected for the laser energy fluctuations. The atomic nitrogen lines detected have been listed in Table 1 with the line data obtained from the NIST database [20]. The average spectrum calculated from the 100 measured spectra are presented in figures 5 and 6.

The influence of gas pressure on the 6 detected lines is shown in Fig. 5 at fixed gas temperatures of $25{ }^{\circ} \mathrm{C}, 100{ }^{\circ} \mathrm{C}$ and $200{ }^{\circ} \mathrm{C}$. For a fixed gas temperature the line intensity decreases with pressure. It should also be noted that the effect of pressure is similar for all nitrogen lines. Figure 6 shows the effect of gas temperature on the N(I) line intensities at a fixed pressure. For a fixed gas pressure the N(I) line intensities increase with the gas temperature. In the three pressure cases shown in Fig. 6 the line intensities are the strongest for $200{ }^{\circ} \mathrm{C}$. The lines are also broader for the $200{ }^{\circ} \mathrm{C}$ case. These results show that there is a definite influence of the gas density (variation of gas pressure and temperature) on the plasma and the detected signal. The ability to detect $\mathrm{N}(\mathrm{I})$ lines at gas pressure of $20 \mathrm{bar}$ and temperature of $200{ }^{\circ} \mathrm{C}$ shows that the LIBS probe with the special optical configuration can be used.

To test the reproducibility and if the data is physically reasonable, an attempt to deduce the mean plasma temperature was made using the mean spectrum shown in Fig. 5 and 6. Even if using close lines is not recommended to achieve meaningful plasma temperatures, it should be possible to see if the trend is linked physically as expected. All the data was processed using a Matlab script written specifically for these cases. First of all the six lines given in Table 1 were separated by setting the limits of the end points for each line and the center wavelength being the values given in Table 1. The integrated signal from a straight line between the end points was used as the background. This value was subtracted from the signal. Using the curve fit toolbox from Matlab a fit for each line was obtained using the multiple-term Gaussian library model.

Figure 7 shows the corrected data points of all six lines as well as the curve fits for the minimum and maximum gas pressure and temperature. As shown all the lines at 1 bar and $25^{\circ} \mathrm{C}$ have high peak intensities and hence curve fitting these data points has been simple. Since the purpose of the LIBS probe is to perform measurements at pressures around 20 bar and temperatures above $200{ }^{\circ} \mathrm{C}$, the question remains how good the curve fits will be at lower signal intensities. As seen in Fig. 7 the measured data points from the nitrogen lines at 20 bar and $200{ }^{\circ} \mathrm{C}$ can be well represented by these curve fits. In case of measurements performed at gas temperatures higher than $200{ }^{\circ} \mathrm{C}$ the line strength should be stronger as the density will decrease and thus this will allow better curve fits.

Assuming a Maxwell-Boltzmann distribution for the energy distribution function of the ${ }^{4} \mathrm{P}-$ ${ }^{4} \mathrm{~S}^{o}$ transition at $746.831 \mathrm{~nm}$ and ${ }^{4} \mathrm{P}-{ }^{4} \mathrm{P}^{o}$ at $824.239 \mathrm{~nm}$ henceforth these two lines are chosen for the plasma temperature calculation. The two lines chosen are well isolated and have minimum interference from any of the neighboring lines. The plasma temperature is determined using Eqn.1, where I is the line intensity, $\lambda$ the wavelength, $g$ is the statistical weight of the level, A is the absorption oscillator strength for the transition, $\mathrm{E}$ is the energy of the upper level, $\mathrm{k}$ is the 
Boltzmann constant and $\mathrm{T}$ is the plasma temperature. The subscripts 1 and 2 represent the two lines. Using the integrated signal from the curve fits and the data provided in Table 1 the mean plasma temperature is calculated.

$$
\frac{I_{1}}{I_{2}}=\frac{\lambda_{2} g_{1} K_{1}}{\lambda_{1} g_{2} K_{2}} \exp \frac{-\left(E_{1}-E_{2}\right)}{k T}
$$

Figure 8 a) shows influence of gas pressure and temperature on the mean plasma temperature calculated from the ratios of the intensities from lines at $746.831 \mathrm{~nm}$ and $824.239 \mathrm{~nm}$. It should be noted that the average laser energy for this measurement was $250 \mathrm{~mJ}$ and the signal was collected in the back scatter mode. The mean plasma temperature decreases as the pressure increases and this could be due the strength and size of plasma as shown earlier in Fig.4. The error in the temperature calculated using the two line method can be around $10 \%$ at best of measuring conditions. In the current measurement the error is approximately $20 \%$ based on the accuracy of the calibration and the fluctuations in the measured spectrum resulting from the hardware, as well as the correction of the background. The error is quite high as expected when using such close lines.

In order to compare the trends shown in the data obtained from the donut beam setup, a second set of measurement was performed using a round laser beam profile and collecting the plasma signal at $90^{\circ}$ angle through one of the access windows available in the high pressure cell. Figure $8 \mathrm{~b}$ ) shows the decay in plasma temperature with pressure similar to what is seen with measurements performed using the probe with donut beam optics. It should be noted that the higher temperatures in Fig. 8 b) could be due to the high laser energy. The influence of gas temperature seems to be small compared to the pressure effect. At 1 bar the plasma temperature ranges from $7400 \mathrm{~K}$ to $8900 \mathrm{~K}$ as measured using the LIBS probe and this temperature decays to range of $2400 \mathrm{~K}$ to $3200 \mathrm{~K}$ at gas pressure of 20 bar. The plasma temperature is an important parameter for determining the concentration of species present in a sample. Therefore the plasma temperature obtained from the measurements done using the LIBS probe provides confidence in testing the LIBS probe at the high pressure rig and later performing measurements at the gasifier facility under high pressure and temperature environment.

\section{Conclusion}

A probe has been designed and developed to perform laser induced breakdown spectroscopy measurements in gas at high pressure and temperature. A set of special optics is used for transforming a round beam profile into a donut shaped beam profile which is then transmitted through the probe to form plasma and the signal from the plasma is collected in the back scatter mode using a mirror mounted in the center of the donut beam profile. The transmission of laser beam and collection of plasma signal through the LIBS probe has been tested using a high pressure cell filled with nitrogen gas where the gas pressure has been varied between 1 to 20 bar at 3 different gas temperatures ranging from 25 to $200{ }^{\circ} \mathrm{C}$.

The plasma imaged on a CCD camera shows the influence of gas pressure and temperature on the shape and size of the plasma from a donut and a round beam profile. The changes in the gas density due to variation of pressure and temperature reduce the size of the focal volume where the plasma is formed. The line intensities measured using the LIBS probe at high pressure and high temperature are high enough to be easily delineated. Mean plasma temperature calculated using the ratio of intensities from lines $746.831 \mathrm{~nm}$ and $824.239 \mathrm{~nm}$ show reasonable values. 
Even though the errors involved in the temperature may be considered high the trend shown is one that is expected.

A good agreement is seen between the decay trend of the plasma temperatures obtained using a round laser beam with signal collection at $90^{\circ}$ to the laser beam axis and the donut laser beam with signal collection in back scatter. This provides confidence in further developing the donut beam optics in combination with the LIBS probe for measurements to be performed at a high pressure test facility.

\section{Acknowledgments}

The work presented here has been carried out as part of the TEIMAB project (SFG Vergasungsreaktor mit Teilgnench und Abhitzesystem, 0327797D) which is funded by the German Federal Ministry of Economics and Technology and Siemens AG. The authors take the responsibility for the content of the paper. The authors would also like to acknowledge all the help provided by Mr Tobias Raap in setting up of the experiments.

\section{References}

[1] McKinsey \& Company, available at <http://www.mckinsey.com/clientservice/ccsi/pdf/CCS_Assessing the_Economics.pdf>

[2] J. Rezaiyan, N. P. Cheremisinoff, Gasification Technologies A Primer for Engineers and Scientists, Taylor \& Francis, 2005.

[3] D. A. Cremers, L. J. Radziemski, Handbook of Laser-Induced Breakdown Spectroscopy, Wiley, New York, 2006.

[4] D. A. Cremers, R. C. Chinni, Laser-Induced Breakdown Spectroscopy-Capabilities and Limitations, Appl. Spectrosc. Rev. 44 (2009) 457-506.

[5] D. W. Hahn, N. Omenetto, Laser-Induced Breakdown Spectroscopy (LIBS), Part I: Review of Basic Diagnostics and Plamsa-Particle Interactions: Still-Challenging Issues With in the Analytical Plasma Community, Appl. Spectrosc. 64 (12) (2010) 335-362

[6] L. Dudragne, Ph. Adam, J. Amouroux, Time-Resolved Laser-Induced Breakdown Spectroscopy: Application for Qualitative and Quantitative Detection of Fluorine, Chlorine, Sulfur, and Carbon in Air, Appl. Spectrosc. 52 (10) (1998) 1321-1327.

[7] H. Zhang, F.-Y. Yueh, J. P. Singh, Laser-induced breakdown spectrometry as a multimetal continuous-emission monitor, 38 (9) (1999) 1459-1466.

[8] K. E. Eseller, F.-Y. Yueh, J. P. Singh, Non-intrusive, on-line, simultaneous multi-species impurity monitoring in hydrogen using LIBS.

[9] V. Sturm, R. Noll, Laser-induced breakdown spectroscopy of gas mixtures of air, $\mathrm{CO}_{2}, \mathrm{~N}_{2}$, and $\mathrm{C}_{3} \mathrm{H}_{8}$ for simultaneous C, H, O, and N measurement, Appl. Opt. 42 (30) (2003) 6221-6225.

[10] L. Zimmer, S. Tachibana, Laser induced plasma spectroscopy for local equivalence ratio measurements in an oscillating combustion environment, Proc. Combust. Inst. 31 (2007) 737-745.

[11] T.-W. Lee, N. Hegde, Laser-induced breakdown spectroscopy for in situ diagnostics of combustion parameters including temperature, Combust. Flame 142 (2005) 314-316.

[12] L. G. Blevins, C. R. Shaddix, S. M. Sickafoose, P. M. Walsh, Laser-induced breakdown spectroscopy at high temperatures in industrial boilers and furnaces, Appl. Opt. 42 (30)(2003) 6107-6118.

[13] A. Molina, P. M. Walsh, C. R. Shaddix, S. M. Sickafoose, L. G. Blevins, Laser-induced breakdown spectroscopy of alkali metals in high-temperature gas, Appl. Opt. 45 (18)(2006) 4411-4423.

[14] M. Kurihara, K. Ikeda, Y. Izawa, Y. Deguchi, H. Tarui, Optimal boiler control through real-time monitoring of unburned carbon in fly ash by laser-induced breakdown spectroscopy, Appl. Opt. 42 (30)(2003) 6159-6165.

[15] C. E. Romero, R. De Saro, J. Craparo, A. Weisberg, R. Moreno, Z. Yao, Laser-Induced Breakdown Spectroscopy for Coal Characterization and Assessing Slagging Propensity, Energy Fuels 24 (2010) 510-517.

[16] C. E. Dumitrescu, P. V. Puzinauskas, S. Olcmen, Movable fiber probe for gas-phase laser-induced breakdown spectroscopy, Appl. Opt. 47 (31) (2008) 88-98.

[17] C. E. Dumitrescu, P. V. Puzinauskas, S. Olcmen, S. G. Buckley, S. Joshi. A. P. Yalin, Fiber-Optic Spark Delivery for Gas-Phase Laser-Induced Breakdown Spectroscopy, Appl. Spectrosc. 61 (12) (2007) 1338-1343. 
[18] B. K. Kapadia, P. Kutne, J. D. Gounder, W. Meier, Combustion behavior of swirl stabilised oxyfuel flames at elevated pressure, 9th AIAA Annual International Energy Conversion Engineering Conference, Papernr AIAA 2011-5593, San Diego, USA, 2011.

[19] K.Loebe, A. Uhl, H. Lucht, Microanalysis of tool steel and glass with laser-induced breakdown spectroscopy, Appl. Opt. 42 (30) (2003) 6166-6173.

[20] P.J. Linstrom, W.G. Mallard, Eds., NIST Chemistry WebBook, NIST Standard Reference Database Number 69 , National Institute of Standards and Technology, Gaithersburg MD, 20899, <http://webbook.nist.gov, (retrieved December 13, 2011). 
Table 1: N(I) line data of the detected lines. The data was obtained from the NIST database [20].

\begin{tabular}{lccc}
\hline$\lambda \mathrm{nm}$ & $\mathrm{g}_{k} \mathrm{~A}_{k} i\left(10^{8} \mathrm{~s}^{\mathrm{I}}\right)$ & $\mathrm{E}_{k}(\mathrm{eV})$ & $\mathrm{g}_{i}-\mathrm{g}_{k}$ \\
\hline 742.364 & 0.226 & 11.9955 & $2-4$ \\
744.229 & 0.476 & 11.9955 & $4-4$ \\
746.831 & 0.784 & 11.9955 & $6-4$ \\
821.634 & 0.226 & 11.8444 & $6-6$ \\
822.314 & 0.262 & 11.8374 & $4-2$ \\
824.239 & 1.31 & 11.7397 & $6-4$ \\
\hline
\end{tabular}


Figure captions:

Fig. 1 Sketch of high pressure LIBS measurement probe. b) LIBS probe mounted on the high pressure rig at DLR.

Fig. 2 High pressure and temperature LIBS experimental setup.

Fig. 3 Density variation of nitrogen gas due to changing pressure and temperature.

Fig. 4 a) Images of plasma formed using donut beam profile and b) plasma formed using round laser beam profile at gas temperatures of $25^{\circ} \mathrm{C}, 100{ }^{\circ} \mathrm{C}, 200^{\circ} \mathrm{C}$ and gas pressures of $1,5,10,15$ and 20 bar.

Fig. 5 Effect of gas pressure on nitrogen line strength measured at 3 gas temperatures.

Fig. 6 Effect of gas temperature on nitrogen line strength measured at 3 gas pressures.

Fig. 7 Corrected data points and curve fit for the measured lines of nitrogen at gas pressures 1 and 20 bar and temperatures $25^{\circ} \mathrm{C}$ and $200{ }^{\circ} \mathrm{C}$.

Fig. 8 a) Influence of gas pressure and temperature on mean plasma temperature obtained from measurements performed using the LIBS probe with donut beam profile (Laser energy $\approx 250$ $\mathrm{mJ}$ ) and back scatter collection. b) Mean plasma temperature calculated from measurements performed using a round laser beam profile (Laser energy $\approx 320 \mathrm{~mJ}$ ) and signal collection at $90^{\circ}$. 

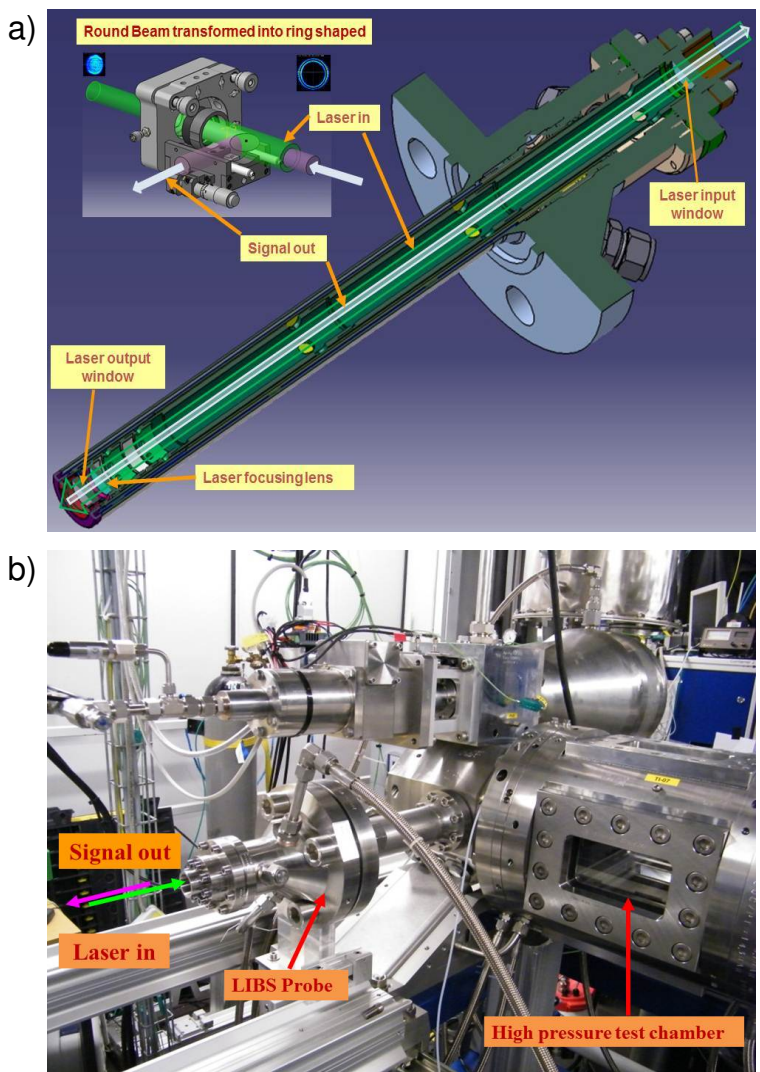

Figure 1: a) Sketch of high pressure LIBS measurement probe. b) LIBS probe mounted on the high pressure rig at DLR. 


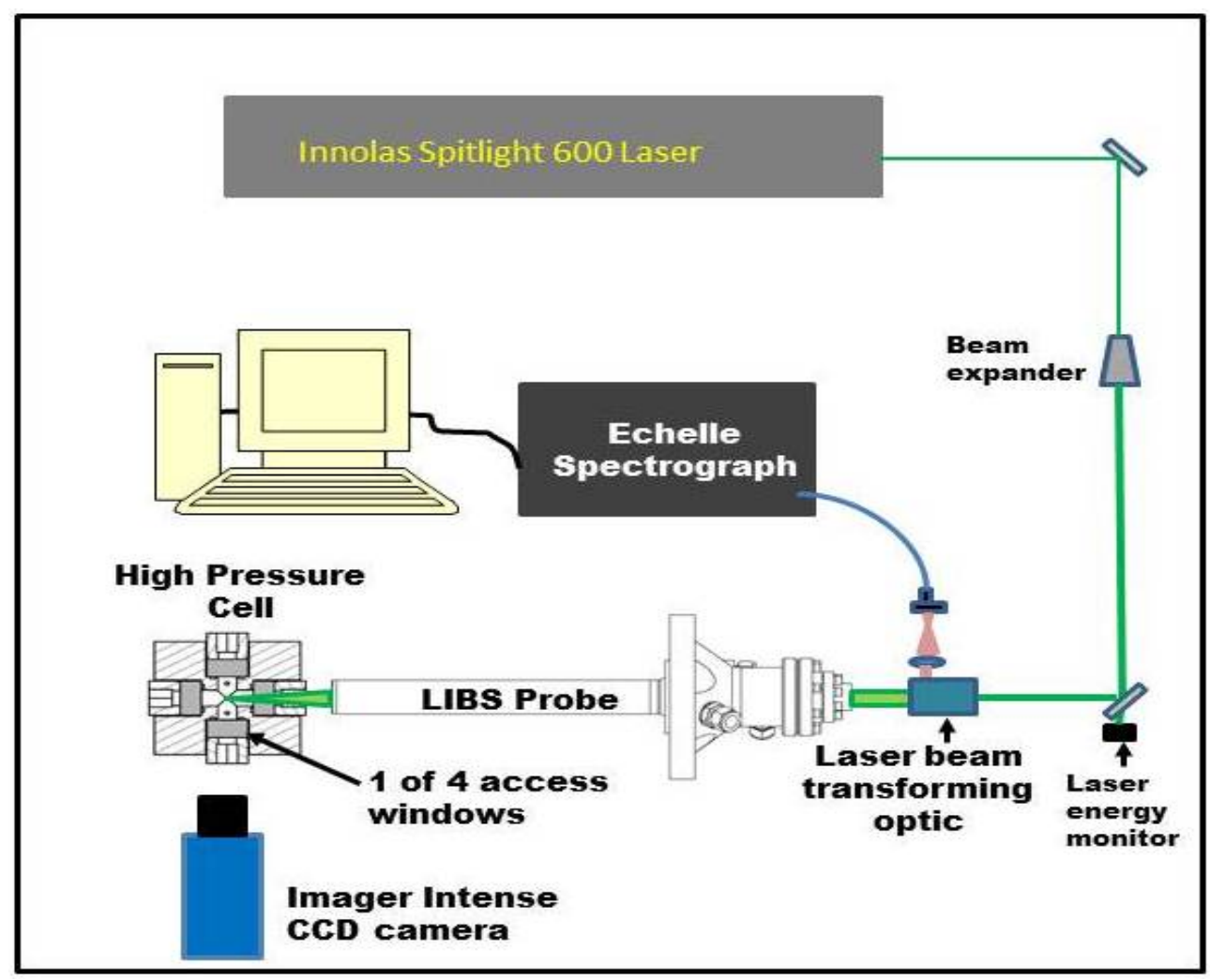

Figure 2: High pressure and temperature LIBS experimental setup. 


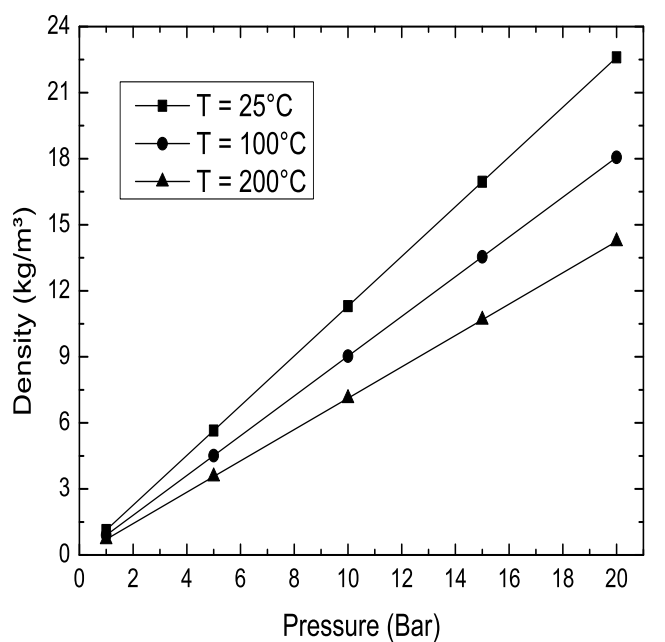

Figure 3: Density variation of nitrogen gas due to changing pressure and temperature. 


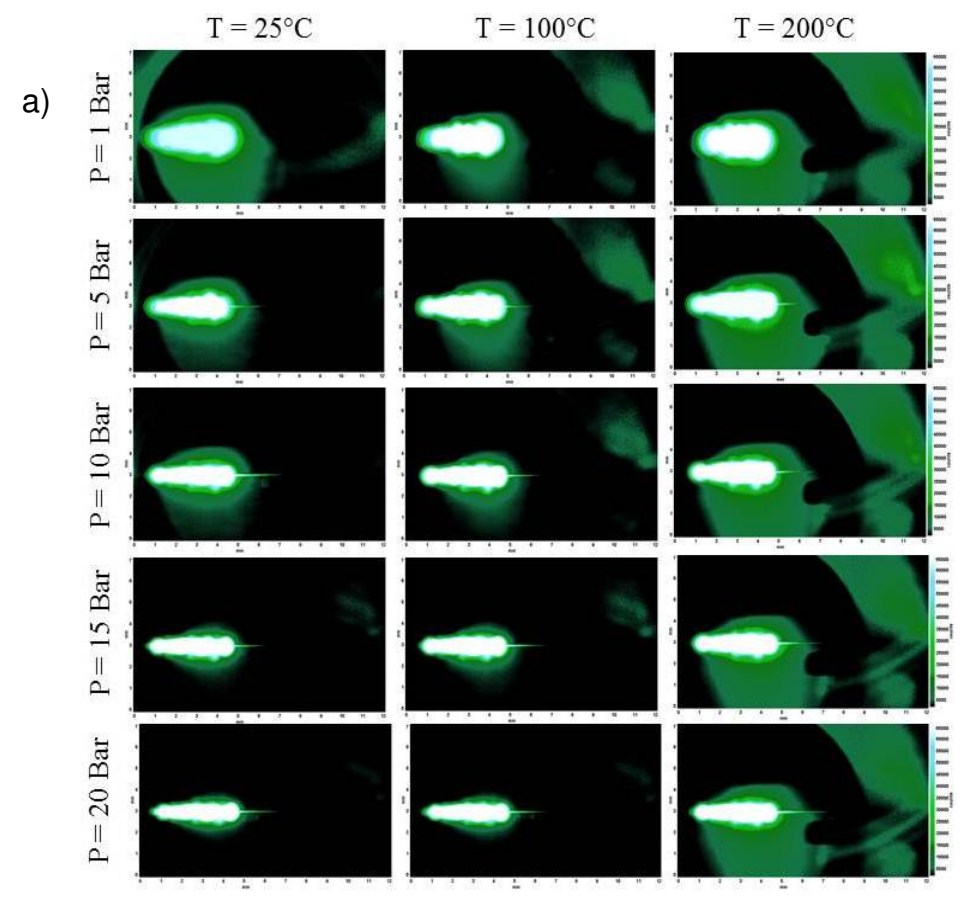

b)
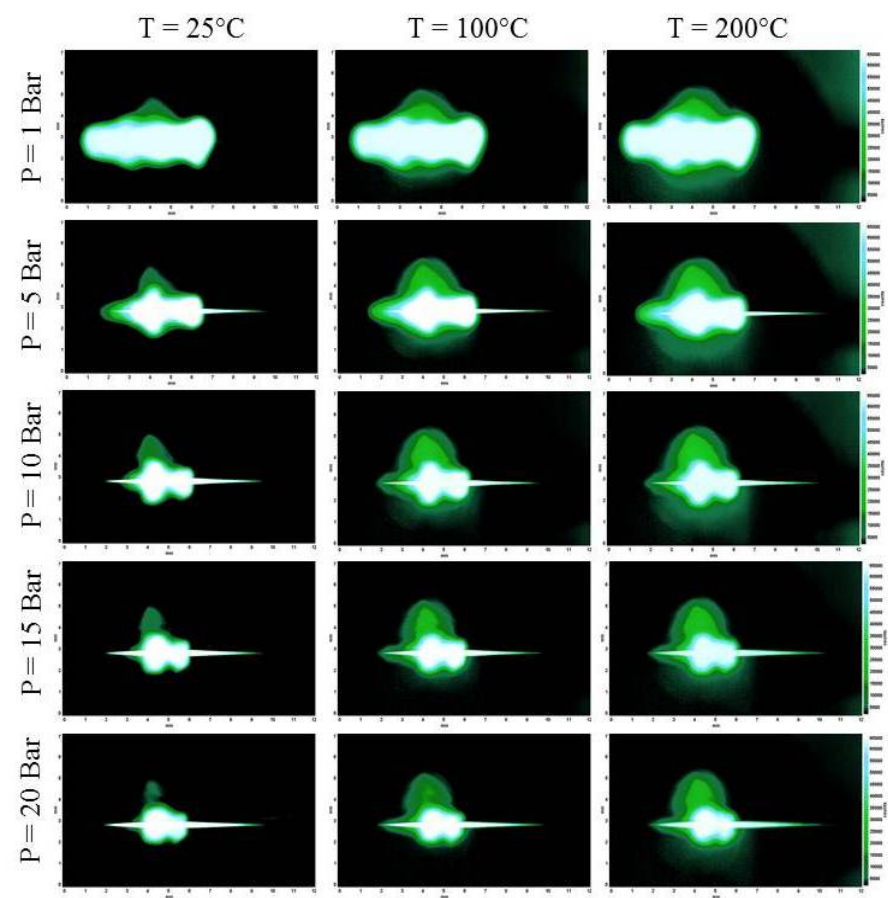

Figure 4: a) Images of plasma formed using donut beam profile and b) plasma formed using round laser beam profile at gas temperatures of $25^{\circ} \mathrm{C}, 100^{\circ} \mathrm{C}, 200^{\circ} \mathrm{C}$ and gas pressures of $1,5,10,15$ and 20 bar. 

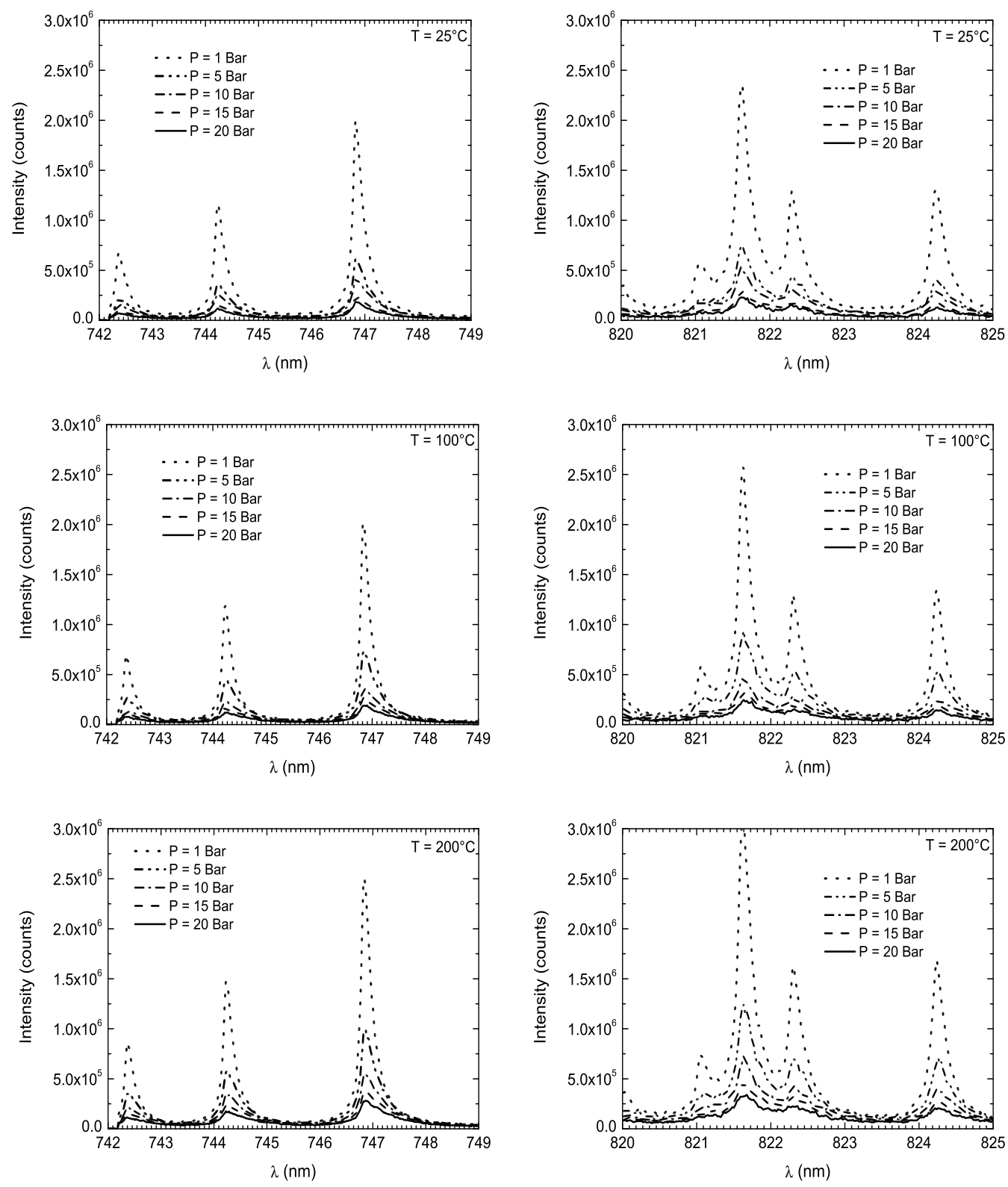

Figure 5: Effect of gas pressure on nitrogen line strength measured at 3 gas temperatures. 

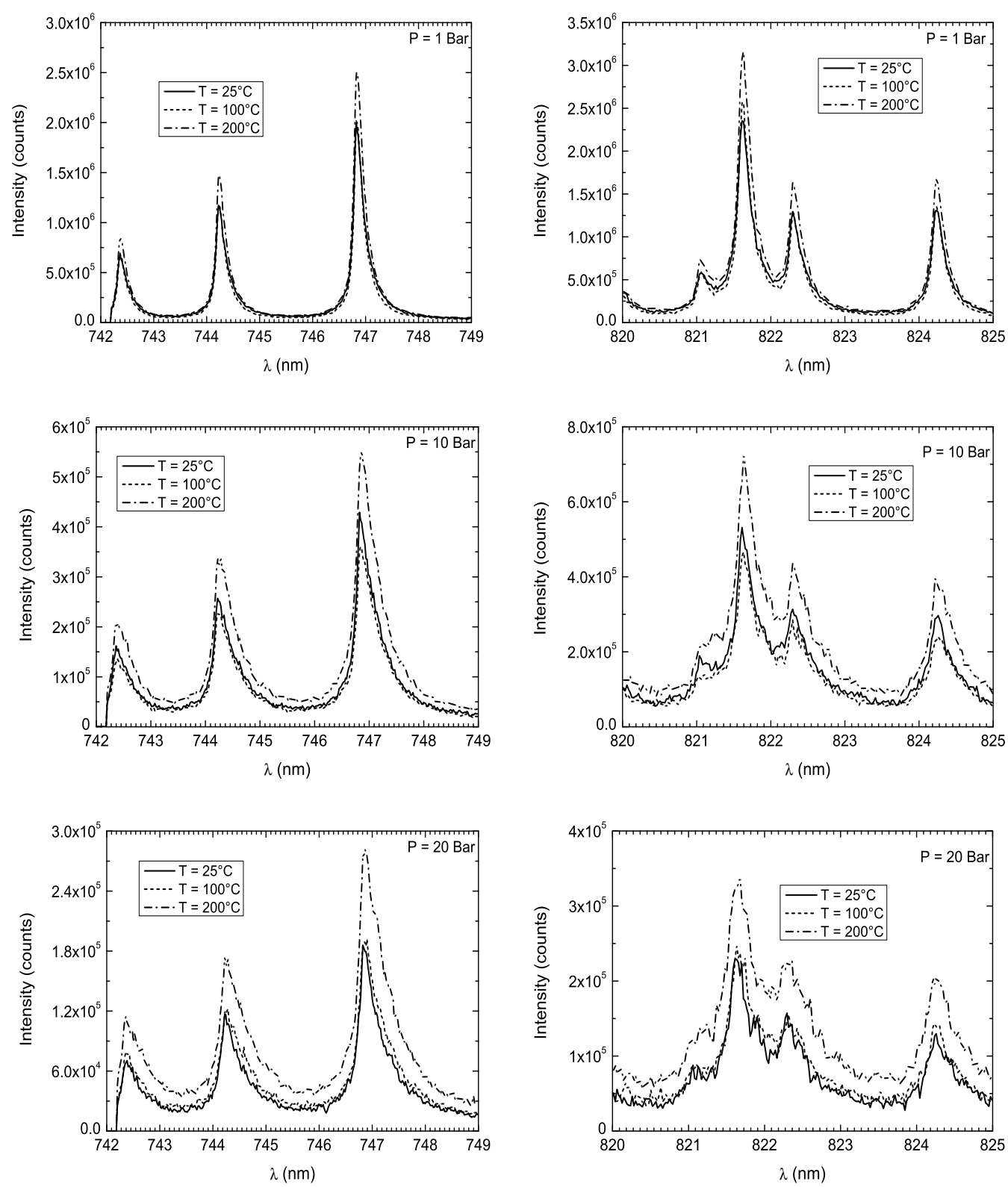

Figure 6: Effect of gas temperature on nitrogen line strength measured at 3 gas pressures. 

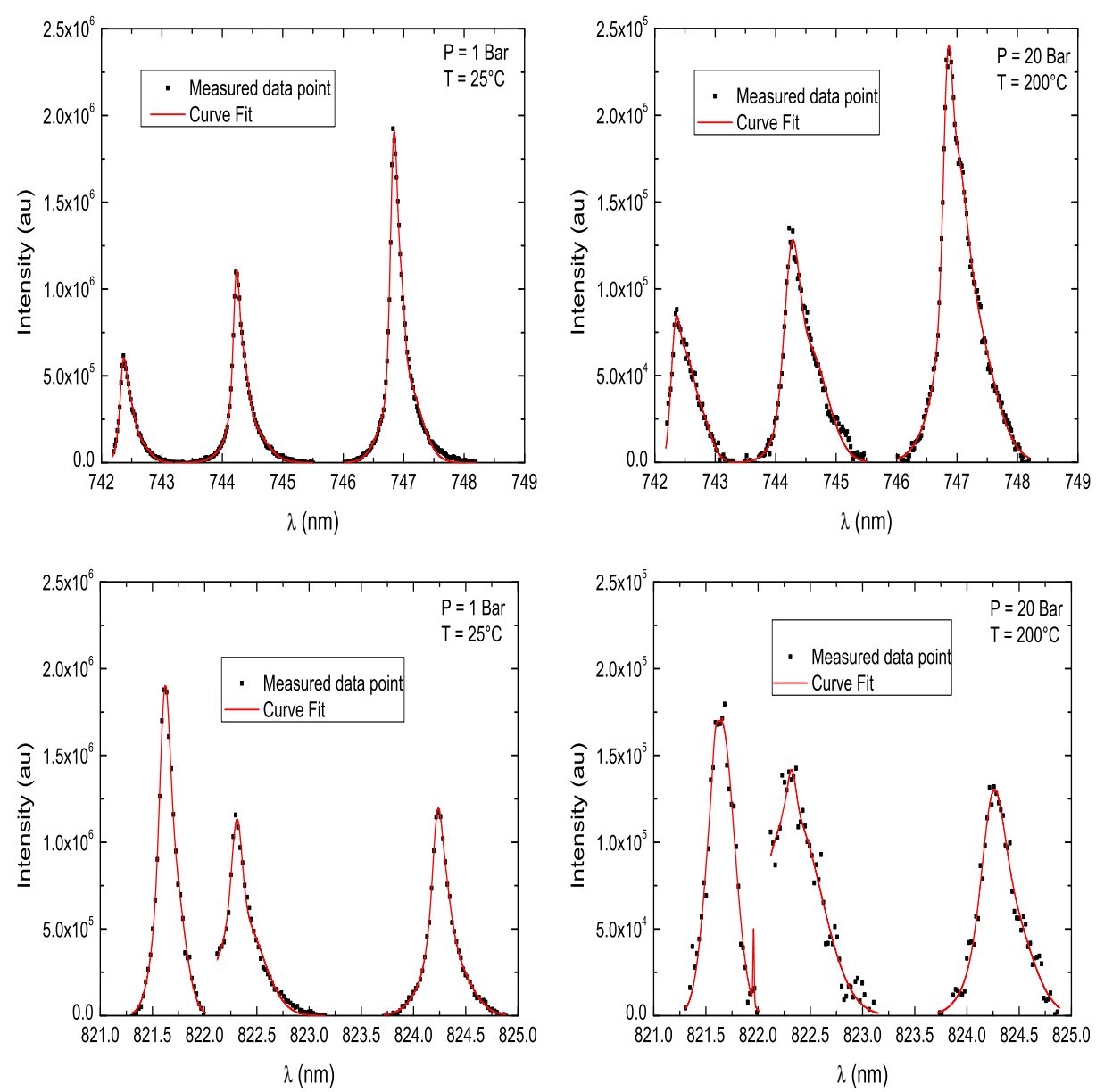

Figure 7: Corrected data points and curve fit for the measured lines of nitrogen at gas pressures 1 and 20 bar and temperatures $25^{\circ} \mathrm{C}$ and $200^{\circ} \mathrm{C}$. 
a)

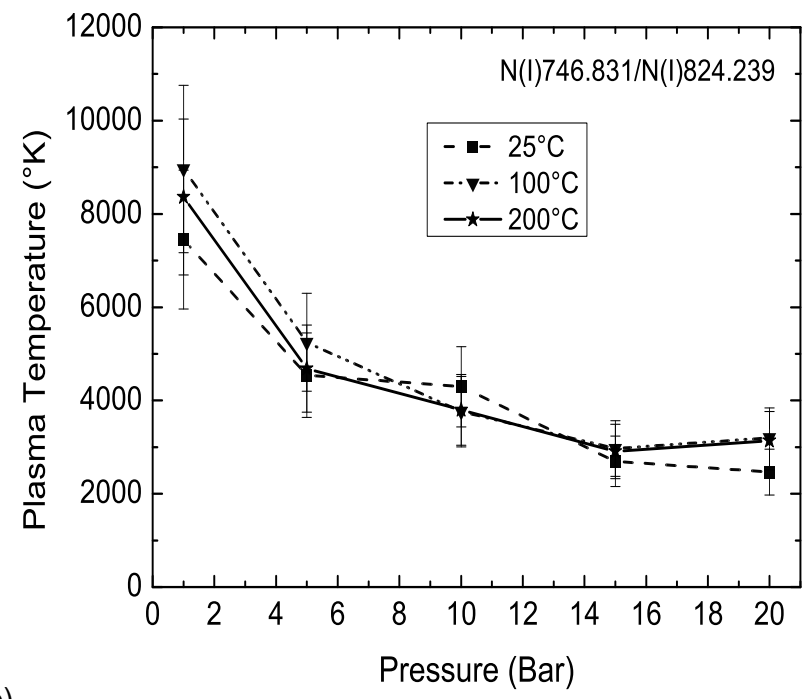

b)

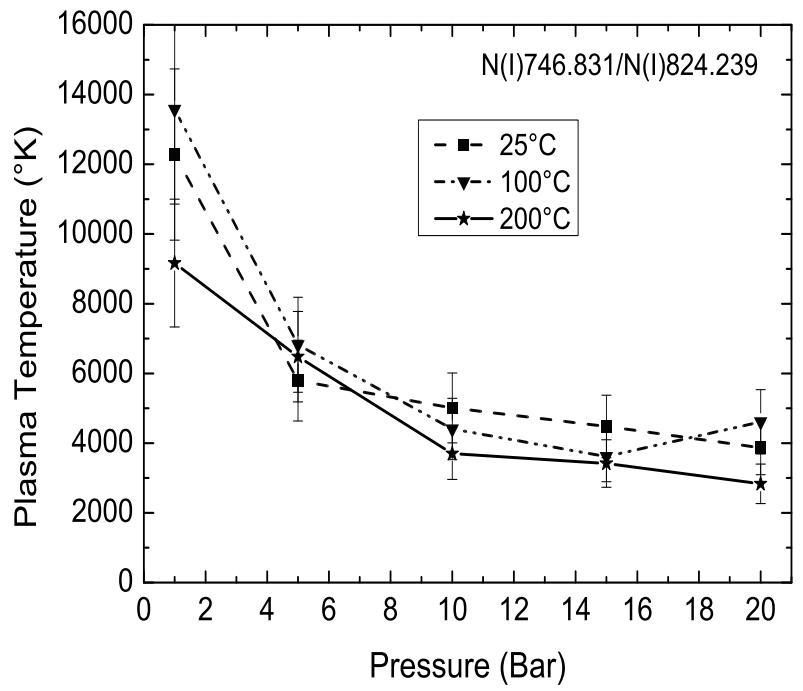

Figure 8: a) Influence of gas pressure and temperature on mean plasma temperature obtained from measurements performed using the LIBS probe with donut beam profile (Laser energy $\approx 250 \mathrm{~mJ}$ ) and back scatter collection. b) Mean plasma temperature calculated from measurements performed using a round laser beam profile (Laser energy $\approx 320 \mathrm{~mJ}$ ) and signal collection at $90^{\circ}$. 